\title{
DETECÇÃO DOS TRANSTORNOS INVASIVOS NA CRIANÇA: PERSPECTIVA DAS MÃES
}

\author{
Maria Rita Braga ${ }^{2}$ \\ Lazslo Antonio Ávila ${ }^{3}$
}

Braga MR, Ávila LA. Detecção dos transtornos invasivos na criança: perspectiva das mães. Rev Latino-am Enfermagem 2004 novembro-dezembro; 12(6):884-9.

Os Transtornos Invasivos do Desenvolvimento se caracterizam por alterações presentes desde idades precoces e se manifestam nas áreas de desenvolvimento da comunicação, comportamento e relação interpessoal. $O$ objetivo deste estudo foi apreender, através da perspectiva materna, como se deu o processo de detecção desses transtornos. Para tanto, realizouse um estudo quanti-qualitativo a partir de entrevista semi-estruturada com 20 mães de crianças portadoras desses quadros. $A$ classificação se deu por meio de análise de conteúdo. Os resultados indicaram que o diagnóstico desses transtornos está sendo realizado após a idade preconizada e que os primeiros sinais característicos são percebidos pela família, principalmente, pela mãe. Esses resultados sugerem que os profissionais de saúde infantil parecem não estar sensibilizados para a importância do efetivo acompanhamento do desenvolvimento, o que permitiria a detecção precoce desses desvios, minimizando, assim, seus efeitos negativos presentes e futuros.

DESCRITORES: desenvolvimento infantil; crianças portadoras de deficiência; diagnóstico precoce

\section{DETECTION OF PERVASIVE DISORDERS IN THE CHILD: A MATERNAL PERSPECTIVE}

Invasive Developmental Disorders are characterized by the early occurrence of changes that come up in the areas of communication development, behavior and interpersonal relationship. This study aimed to discover how the disorder detection process took place from a maternal perspective. A quantitative and qualitative study was carried, based on a semi-structured survey with 20 mothers of children in this condition. Results were processed through content analysis and indicated that these disorders have been diagnosed after the established age and that early typical signs are noticed by the family, particularly by the mother. These findings that child health professionals do not seem to be aware of the importance of effective developmental follow-up, which would lead to the early detection of these disorders, thus minimizing its present and future negative effects.

DESCRIPTORS: child development; disabled children; early diagnosis

\section{DETECCIÓN DE LOS TRASTORNOS INVASIVOS EN EL NIÑO: PERSPECTIVA DE MADRES}

Los Trastornos Invasivos del Desarrollo se caracterizan por alteraciones presentes en edades precoces y se manifiestan en las áreas de desarrollo de la comunicación, comportamiento y relación interpersonal. El objetivo de este estudio ha sido aprender a través de la perspectiva materna como ocurrió el proceso de detección de estos trastornos. Para eso, se realizó un estudio cuanti-cualitativo a partir de una encuesta semi-estructurada con 20 niños portadores de estos cuadros. La clasificación fue realizada a través de análisis de contenido. Los resultados indicaron que el diagnóstico de estos trastornos está siendo realizado tras la edad preconizada y que las primeras señales características son percibidas por la familia, principalmente por la madre. Estos resultados sugieren que los profesionales de salud infantil parecen no estar sensibilizados para la importancia del efectivo acompañamiento del desarrollo, lo que permitiría la detección temprana de estos desvios minimizando, así, efectos negativos presentes y futuros.

DESCRIPTORES: desarrollo infantil; niños con discapacidad; diagnóstico temprano

\footnotetext{
${ }^{1}$ Trabalho extraído da dissertação de mestrado apresentada à Faculdade de Medicina de São José do Rio Preto; ${ }^{2}$ Enfermeira, Mestranda da Faculdade de Medicina de São José do Rio Preto, Docente da Faculdade de Enfermagem de Catanduva, e-mail: mariaritabr@yahoo.com.br; ${ }^{3}$ Psicólogo, Pós-Doutorado pela Universidade de Cambridge (Inglaterra), Professor Adjunto da Faculdade de Medicina de São José do Rio Preto, e-mail: lazslo@terra.com.br
} 
INTRODUÇÃO

Os Transtornos Invasivos do Desenvolvimento, também denominados transtornos globais do desenvolvimento, constituem um grupo caracterizado por alterações presentes desde idades precoces e que se manifestam nas áreas de desenvolvimento da comunicação, comportamento e relação interpessoal ${ }^{(1)}$.

Essas alterações são constatadas quando há atraso em relação ao esperado para uma determinada idade ou estágio de desenvolvimento da criança.

Compondo tal grupo temos: o transtorno autístico, o transtorno de Asperger, a síndrome de Rett, o transtorno desintegrativo infantil e transtornos invasivos do desenvolvimento não especificados de outra forma ${ }^{(1)}$.

Entre eles, o autismo é o mais complexo. As crianças autistas apresentam uma forma atípica de se desenvolver, mantendo sempre uma expressão facial de distanciamento, o que dá a seus interlocutores a sensação de que estão interagindo com alguém que pertence a um outro mundo.

"Estas crianças desenvolvem uma carapaça, uma concha, que as protege do mundo exterior e também atua como uma barreira de acesso ao mundo"(2).

Além dessas características, o autista apresenta movimentos repetitivos e estereotipados, como o torcer as mãos ou os dedos e uma inflexibilidade em relação a alterações nas rotinas. $O$ autismo também é o mais preponderante entre esses transtornos e parece estar aumentando em freqüência nos estudos epidemiológicos recentes.

Segundo a literatura ${ }^{(1)}$, em 1995 existiam 4 a 5 casos para cada 10000 nascimentos. Já em 1999, utilizando critérios diagnósticos mais abrangentes, a estimativa atinge cerca de um afetado para cada 1000 nascimentos $^{(3)}$.

Recentes estudos sugerem que até 1 em cada 150 crianças com dez anos ou menos podem ser afetadas pelo autismo ou desordens relacionadas - um total de cerca de 300 mil crianças somente nos Estados Unidos. Se incluir os adultos, segundo a Autism Society of América, mais de um milhão de pessoas nos Estados Unidos sofrem de um dos transtornos invasivos do desenvolvimento. O problema é cinco vezes mais comum que a síndrome de Down e três vezes mais freqüente que a diabete juvenil $^{(4)}$.

Paradoxalmente, o mesmo fato que é tão grave nas desordens autistas - afetam as crianças muito pequenas - também sugere motivo para esperança, pois como as conexões neurais no cérebro de uma criança são estabelecidas por meio da experiência, exercícios mentais e estímulos ao desenvolvimento cognitivo e emocional bem planejados podem potencialmente trazer transformações ${ }^{(4)}$. Justifica-se assim a necessidade de detecção precoce desses transtornos e o encaminhamento da criança para confirmação diagnóstica e tratamento.

Além da observação clínica da criança, existem vários testes auxiliares para se avaliar e acompanhar o desenvolvimento infantil, entre eles o teste de triagem de desenvolvimento de Denver ${ }^{(5)}$ e, mais especificamente para detectar sinais característicos de autismo, a Ficha de Acompanhamento do Desenvolvimento Infantil ${ }^{(6)}$.

O próprio cartão da criança, instrumento distribuído pelo Ministério da Saúde nas unidades básicas, contém, além da curva de crescimento, a ficha de desenvolvimento infantil. Esses instrumentos são os componentes críticos da avaliação completa da criança e têm por finalidade demonstrar como ela se compara com outras crianças da mesma idade.

O enfermeiro, enquanto membro da equipe de profissionais envolvidos no cuidado da saúde infantil, desempenha papel fundamental nos procedimentos de triagem, designados para identificar e avaliar o desenvolvimento da criança $a^{(7)}$.

A compreensão dos achados normais para as crianças em diferentes faixas etárias é crítica para interpretar os resultados da avaliação, atuando na educação e orientação preventiva junto aos pais e também para detectar e registrar possíveis desvios ${ }^{(7)}$. A detecção precoce de transtornos no desenvolvimento permite ao enfermeiro agir diretamente, através de um plano de cuidados, e também encaminhar a criança a uma equipe especializada para confirmação diagnóstica e tratamento.

Entretanto, como se pode observar através da experiência profissional, defronta-se com mães que relatam sua problemática referente à demora no processo diagnóstico. Sabendo que essas crianças apresentam risco de morbidade em longo prazo, essa realidade despertou para a relevância do tema.

Entende-se que esse conhecimento poderá fornecer subsídios para instrumentalizar ações dirigidas a tal segmento populacional, visando a integralidade e qualidade da assistência, baseando-se não somente nas necessidades especiais da criança, mas também nas necessidades do seu cuidador.

Assim, com o objetivo de conhecer, através da 
perspectiva materna, como tem se desenvolvido o processo de detecção dos transtornos invasivos do desenvolvimento, foi realizado o estudo que segue.

\section{CASUÍSTICA E MÉTODO}

Entre março e maio de 2003, foram entrevistadas 20 (vinte) mães de crianças com transtornos invasivos do desenvolvimento, após concordarem em participar da pesquisa e assinarem o termo de consentimento livre e esclarecido, respeitando-se os itens constantes nas diretrizes e normas regulamentadoras de pesquisa em seres humanos.

Esta pesquisa foi submetida à avaliação e aprovada pelo Comitê de Ética e Pesquisa da Faculdade de Medicina de São José do Rio Preto, SP, em 13 de janeiro de 2003, Parecer $n^{\circ}$ 006/2003.

Do total de mães entrevistadas, dez tinham filhos matriculados no núcleo de autistas da APAE, do município de Catanduva e, outras dez, na Escola do Autista do município de São José do Rio Preto, ambos do Estado de São Paulo.

O material utilizado constituiu-se de um roteiro de entrevista, previamente elaborado, para investigação do conhecimento da mãe sobre o diagnóstico do filho e história do acompanhamento médico e de enfermagem do desenvolvimento da criança com transtornos invasivos do desenvolvimento, constando ainda dados de identificação da mãe, criança e escola.

A entrevista foi realizada normalmente nas escolas, mas, em 10 casos, quando havia impossibilidade por parte da mãe, aconteceu na própria residência da mesma. Em ambas as situações foram providenciadas privacidade e suficiente isolamento de ruídos e interferências externas, além de iluminação e acomodações adequadas para a realização das sessões.

As entrevistas processaram-se tranqüilamente, por um período de aproximadamente uma hora, com boa interação entrevistador $x$ entrevistada. Atribui-se esse bom relacionamento aos esclarecimentos ocorridos no primeiro contato. O protocolo de registro foi composto por folha de papel sulfite, lápis e caneta, acompanhado de gravador e fitas cassete.

O uso do gravador deu liberdade ao entrevistador para direcionar toda a sua atenção ao entrevistado. 0 pesquisador solicitava autorização da entrevistada para uso do aparelho e mantinha o mesmo à vista, sobre a mesa.
A classificação dos dados foi feita através da análise do conteúdo. Essa técnica permite identificar elementos com um grau de homogeneidade entre si e que apresentam maior freqüência de repetição ${ }^{(8)}$.

Após releitura do material e organização dos relatos, foi realizada a classificação dos dados, em que se identificou o que era mais freqüente e relevante nas entrevistas, para a elaboração de categorias específicas.

Estabelecido o conjunto de categorias, procedeuse à validação, solicitando a colaboração de dois profissionais da área, para atuarem como juízes e, posteriormente, foram feitas a análise e a descrição estatística dessas categorias.

\section{RESULTADOS}

A média das idades maternas foi de 44,6 anos, tendo como idade mínima 26 e máxima, 72 anos.

A maioria das mães cursou o primeiro grau (60\%), sendo que $25 \%$ não conseguiram completar o segundo grau, $15 \%$ o completaram e outras $15 \%$ iniciaram o terceiro grau, mas não terminaram. Apenas $5 \%$ possuem o terceiro grau completo.

Segundo relato das mães, todas as 20 crianças portadoras de Transtornos Invasivos do Desenvolvimento compartilhavam características autísticas, sendo que $75 \%$ pertenciam ao sexo masculino e $25 \%$ ao sexo feminino.

A família, na maioria das vezes (75\%), percebeu os primeiros sinais da criança com características voltadas para o autismo, sendo que foi a mãe, em $40 \%$ dos casos, quem teve essa percepção. A freqüência com que os profissionais da saúde e educação detectaram esses sinais foi $25 \%$.

Até os três anos de idade, apenas $30 \%$ dessas crianças haviam sido diagnosticadas, sendo essa a idade preconizada pela literatura. A maior porcentagem, $65 \%$, se deu após essa idade. Uma das mães (5\%) não soube precisar a idade da criança no momento do diagnóstico (Figura 1).

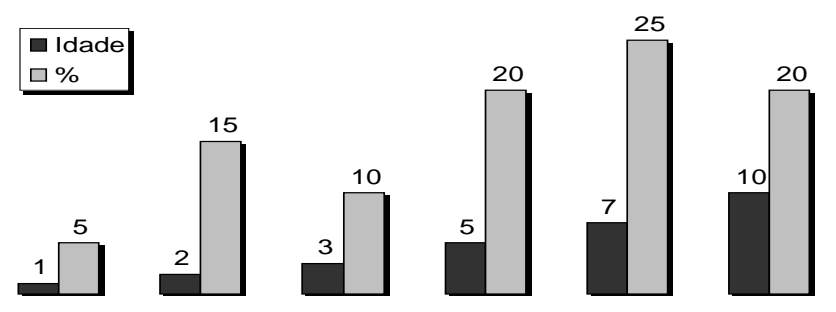

Figura 1 - Freqüência da idade no momento do diagnóstico 
Sobre as etapas do desenvolvimento infantil, 50\% das mães informaram não ter sido utilizado quaisquer instrumentos para tal avaliação, 35\% não souberam dizer se foi ou não utilizado e apenas $15 \%$ responderam positivamente à questão.

Informações sobre esse tema não foram fornecidas pelos profissionais de saúde para $45 \%$ das mães, outras $10 \%$ não lembravam se receberam ou não essas informações e $5 \%$ responderam que receberam "algumas informações, mas não todas".

Nos $40 \%$ dos casos restantes, foi o profissional médico em $30 \%$ das vezes e o psicólogo (10\%), os responsáveis por darem as orientações.

As mães consideram que os profissionais foram adequados ao dar o diagnóstico em $55 \%$ dos casos; foram considerados inadequados em $15 \%$ das vezes e $30 \%$ das mães deram outras respostas a essa questão como, por exemplo: "eu acho que não existe uma maneira adequada".

A grande maioria das mães, 95\%, acredita ser importante saber o diagnóstico do filho e acrescentam que só assim poderão ter uma direção para o tratamento e perspectivas futuras frente à criança.

\section{DISCUSSÃO}

Os dados referentes à prevalência do sexo masculino sobre o feminino na proporção de 3 para 1 é compatível com a literatura referente às crianças que apresentam distúrbios autísticos ${ }^{(9)}$. Isso pode indicar que os transtornos invasivos do desenvolvimento estejam ligados ao cromossomo X, e que sua maior incidência na população masculina também pode estar associada à presença da síndrome do cromossomo $X$ frágil $^{(10)}$.

Os dados referentes à percepção dos sinais característicos dos transtornos invasivos do desenvolvimento mostram que a prevalência da detecção é realizada pela mãe, $40 \%$ dos casos. Essa descreve seu bebê como "estranho", não conseguindo, no entanto, precisar de forma clara esse comportamento, caracterizando-o somente como criança muito quieta, mas com um progressivo surgimento e reconhecimento da sintomatologia $^{(11)}$.

As informações, adquiridas através dos pais, geralmente são confiáveis, devido ao fato de manterem um contato íntimo com a criança ${ }^{(7)}$.

Sabendo que é a mãe quem, naturalmente, percebe os primeiros sinais de distúrbios no desenvolvimento da criança, a enfermeira deve aproveitar todas as oportunidades para ampliar os conhecimentos da mesma, através de orientações e fornecimento de materiais sobre o tema.

É pequena a porcentagem de profissionais da área de saúde que detectam alterações no desenvolvimento infantil, $25 \%$, o que sugere que esses profissionais necessitam desenvolver a capacitação e também sensibilização referente ao tema. É extensa a bibliografia sobre o assunto que orienta sobre a importância dos profissionais conhecerem e avaliarem as etapas do desenvolvimento infantil, para que, conhecendo os padrões de normalidade, possam detectar possíveis desvios.

O motivo pelo qual os diagnósticos, em grande parte, têm sido realizados após a idade preconizada de 3 anos pode estar relacionado às dificuldades encontradas pelos profissionais, devido ao grau variado de severidade desses transtornos ${ }^{(12)}$. Aqui se destaca a importância dos profissionais usarem os testes e escalas de avaliação do desenvolvimento infantil elaborados para auxiliar no diagnóstico.

As mães informam, entretanto, que, na maioria dos casos, esses instrumentos não foram utilizados pelos profissionais ou esses, se os utilizaram, não as informaram.

A literatura alerta o enfermeiro sobre a importância da avaliação do desenvolvimento infantil nos procedimentos de triagem, visto que é ele, freqüentemente, o profissional responsável, nas unidades básicas de saúde e ambulatórios, pela realização das avaliações ${ }^{(7)}$. O enfermeiro deve também informar apropriadamente aos pais sobre essas avaliações, de forma que estejam totalmente cientes da sua finalidade e entregar-lhes uma cópia dos resultados, para que possam ser utilizados por todos que atendam à criança.

A respeito de orientação sobre as etapas do desenvolvimento infantil também é relevante o número de mães que relatam não tê-la recebido dos profissionais, $45 \%$, ou manifestaram dúvidas sobre tal recebimento, $10 \%$, ou, ainda, sobre a qualidade do mesmo, $5 \%$.

Ao trabalhar com crianças, os enfermeiros devem ouvir os pais e considerar suas observações, incluindo-os em seus planos de cuidados, por entender que as relações parentais eficazes dependem do conhecimento dos mesmos sobre o desenvolvimento ${ }^{(7)}$. O enfermeiro também deve assumir seu papel de educador, pois, muitas vezes, é sua a responsabilidade de oferecer elementos 
para que os pais tomem consciência das necessidades da criança.

As mães consideraram que os profissionais foram adequados ao transmitirem o diagnóstico em 55\% dos casos; o que leva a se considerar que possivelmente eles estejam fazendo "aconselhamentos, explicando integralmente a natureza das dificuldades, com orientações objetivas e minorando o medo, além dos sentimentos de culpa e desespero dos pais, enfatizando o fato de que estes últimos não foram os causadores do comportamento

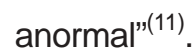

Entende-se, entretanto, que o momento do diagnóstico é muito delicado, pois normalmente ocorre uma "batalha" interna, em que os pais querem saber o que o filho tem, mas não querem acreditar na informação do médico. Nesse caso, os pais sentem necessidade de ouvir essa mesma informação de diversos profissionais, até aceitarem o diagnóstico ${ }^{(13)}$.

O enfermeiro, novamente, pode auxiliar a família, fornecendo apoio e informações através dos dados disponíveis na literatura e relacionados à sua experiência profissional.

A importância dada pela mãe ao diagnóstico é salientada na literatura, quando essa alerta os profissionais para que, só depois do diagnóstico e da identificação de eventuais condições associadas, é possível elaborar um eficiente plano de tratamento e aconselhamento familiar ${ }^{(12)}$.

Além disso, diminuindo a ansiedade dos pais, mediante o conhecimento da doença, eles podem proporcionar melhoras na qualidade de vida desejada ao filho $^{(14)}$.

\section{CONCLUSÕES}

- As mães são as pessoas que mais têm detectado os primeiros sinais característicos dos transtornos invasivos do desenvolvimento, e ela revela ter noção da importância do diagnóstico para o início do tratamento. Os profissionais, porém, ou não estão bem informados ou não estão transmitindo informações adequadas às mães sobre as etapas do desenvolvimento infantil.

- O diagnóstico dos transtornos invasivos do desenvolvimento tem sido realizado após o período preconizado de 3 anos e, no momento do diagnóstico, alguns profissionais, ao participarem os pais, levam em consideração a complexidade e o impacto do mesmo frente à família; enquanto outros não conseguem transmitir o diagnóstico de forma adequada.

\section{CONSIDERAÇÕES FINAIS}

Sabendo que os transtornos invasivos do desenvolvimento manifestam formas graves e que, se não forem detectados e tratados precocemente, tornam-se quadros de difícil manejo, propõe-se que sejam fornecidos à população programas de orientação sobre o assunto, da forma mais atraente e acessível possível, para que as informações sejam bem absorvidas.

Faz-se importante também a publicação freqüente de literatura específica para os profissionais envolvidos com a saúde infantil. Esses últimos necessitam ser sensibilizados e capacitados para o efetivo acompanhamento e avaliação do desenvolvimento infantil. Além da observação clínica, o profissional pode obter auxílio na utilização de tabelas e instrumentos elaborados para esse fim.

Os enfermeiros devem investir nas práticas de atenção à saúde, que promovam o bem-estar da criança a partir da incorporação dos conhecimentos acerca do crescimento e desenvolvimento infantil. Por sua proximidade, inerente à função, junto à díade mãe-filho, deve aproveitar todas as oportunidades de atendimento para potencializar a capacidade da mãe em promover 0 desenvolvimento da criança.

Os profissionais de saúde devem entender que assumem uma posição vital, através da comunicação da sua avaliação para o encaminhamento da criança com transtornos, no desenvolvimento de uma equipe especializada para a confirmação diagnóstica e início do tratamento.

Se confirmado o diagnóstico, novamente o enfermeiro deve estar presente, proporcionando atendimento humanizado, levando em consideração a complexidade e o impacto do diagnóstico na família, além da necessidade dessa ser assistida por um profissional interessado, preparado e que inspire confiança a todos os que convivem com a criança.

O enfermeiro, estando atento a todas as comunicações e expressões de comportamento da criança e dos seus pais, além de ouvi-los, pode ajudá-los a tomar consciência de sua problemática, incentivando-os a examinar as possibilidades e a adequação dos recursos pessoais, familiares, sociais e terapêuticos disponíveis. 
1. APA. Manual diagnóstico e estatístico de transtornos mentais. 4aㅡ ed. Porto Alegre (RS): Artes Médicas; 1995.

2. Ávila LA. Autistas em grupo terapêutico. Temas sobre desenvolvimento 1998 julho; 7(39):36-41.

3. Gilberg C, Wing L. Autism: not an extremely rare disorder. Acta Psychiatr Scand 1999 junho; 99(6):399-406.

4. Nash JM. The secrets of autism. Time 2002 May; 159(18):46-56.

5. Frankenburg WK, Dodds J, Archer P, Shapiro H, Bresnick B. The Denver II: a mayor revision and restandardization of the Denver Developmental Screening Test. Pediatrics 1992; 89(1):91-7.

6. Paravidini JLL. A identificação e o diagnóstico precoce de sinais de risco de autismo infantil. [Tese]. Campinas (SP): Universidade Estadual de Campinas/UNICAMP; 2002.

7. Wong DL. Enfermagem Pediátrica: elementos essenciais à intervenção efetiva. 5aㅡ ed. Rio de Janeiro (RJ): Guanabara; 1997.

8. Turato ER. Tratado da metodologia da pesquisa clínicoqualitativa. Petrópolis (RJ): Vozes; 2003.

9. Jorde LB, Hasstedt SJ, Ritvo ER, Mason-Brothers A, Freeman BJ, Pingree $\mathrm{C}$, et al. Complex segregation analysis of autism. Am J Hum Genet 1991 novembro; 49(5):932-8.

10. Giunco CT. Avaliação genético-clínica e citogenética molecular das regiões 7q31-q33 e 15q11-q13 em transtornos invasivos do desenvolvimento. [Dissertação]. São José do Rio Preto (SP): Instituto de Biociências Letras e Ciências Exatas/UNESP; 2002.

11. Lipp JRS, Torres VM. O perfil multidisciplinar do bebê autista. In: Lipp JRS, Crus AR. Psiquiatria infantil: estudo multidisciplinar. Brasília (DF): Disan; 1987. p.109-20.

12. Schwartzman JS, Assumpção Jr FB. Autismo Infantil. São Paulo (SP): Memnon; 1995.

13. Camargo W Jr. Autismo Infantil. In: Fonseca LF, Pianetti G, Xavier CC. Compêndio de neurologia infantil. Rio de Janeiro (RJ): Medsi; 2002. p.911-18.

14. Furtado MCC, Lima RAG. O cotidiano da família com filhos portadores de fibrose cística: subsídios para a enfermagem pediátrica. Rev Latino-am Enfermagem 2003 jan/fev; 11(1):6673. 